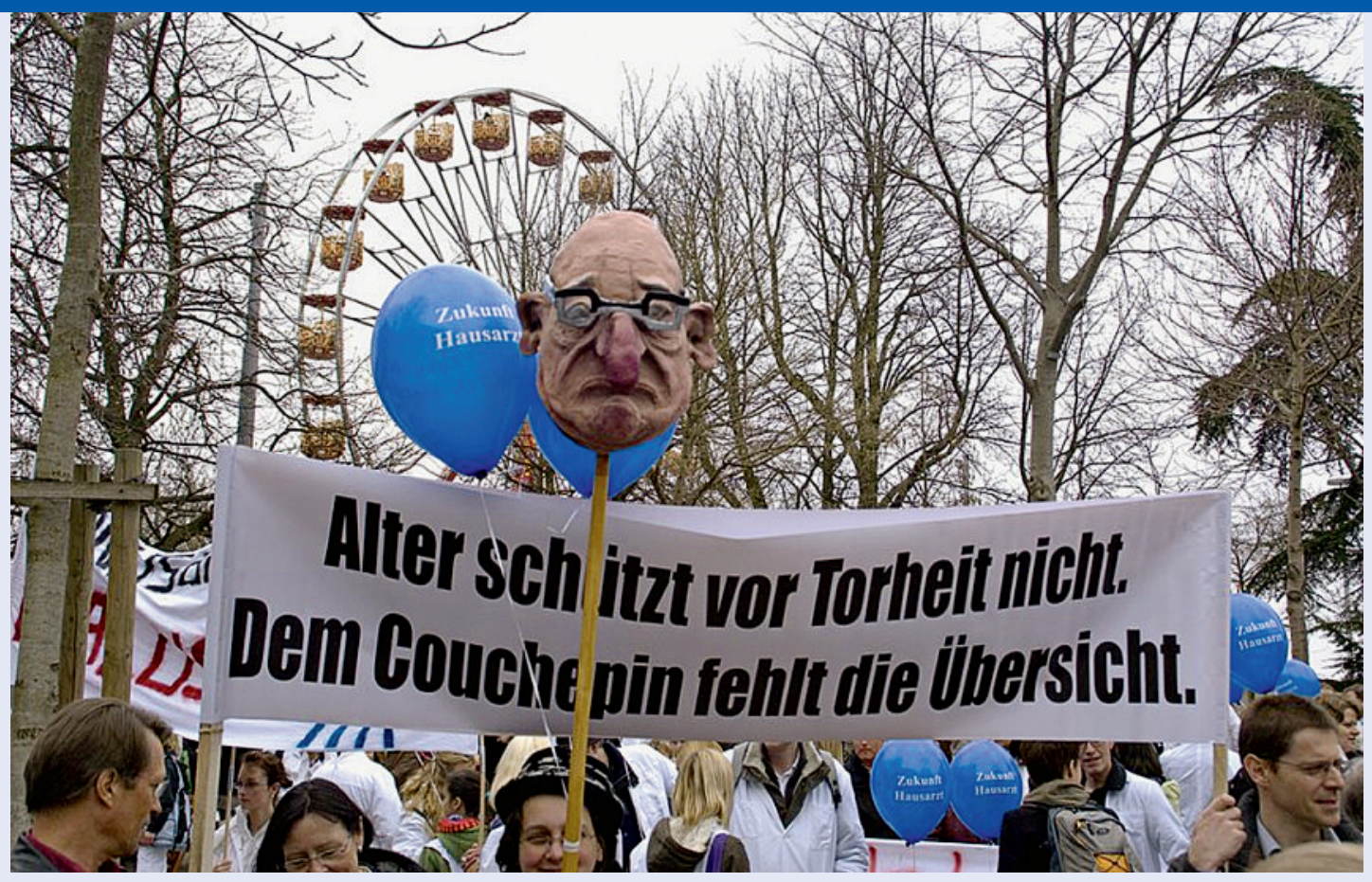

Colère des médecins de famille le 1er avril 2009: une baisse de la marge sur les médicaments frapperait à nouveau

\title{
La FMH s'oppose fermement à une baisse des marges sur les médicaments et demande un dialogue loyal
}

Communication de presse de la FMH du 26 avril 2009

Département Communication

Correspondance:

FMH

Département Communication Elfenstrasse 18

CH-3000 Berne 15

kommunikation@fmh.ch

La FMH proteste avec véhémence contre les citations erronées émanant du procèsverbal rédigé par le Département fédéral de l'intérieur (DFI) au sujet de la table ronde sur les coûts de la santé. Selon le document publié, le président de la FMH, Jacques de Haller, se serait déclaré favorable à une diminution de la marge sur la vente de médicaments. A ce propos, J. de Haller souligne que «la FMH n'a jamais fait et ne fera jamais une telle proposition». En effet, une réduction de cette sorte porterait une nouvelle fois préjudice aux médecins de famille. Le DFI a publié ce procès-verbal sans en informer les principaux intéressés. En agissant de la sorte, le DFI ne fait que rajouter de l'huile sur le feu - au détriment des intérêts en cause dans les discussions déjà fort tendues entre les différents acteurs de la santé.

Le fait que l'hôte de la table ronde, le ministre de la santé Pascal Couchepin, publie un procès-verbal comportant des affirmations erronées - voire trompeuses sans en informer les principaux intéressés, nuit gravement au sens et au but des débats qui ont eu lieu. La façon dont l'administration fédérale tourne le dos à la culture du dialogue illustre la pression accrue qui s'exerce sur les protagonistes de la santé. Agir ainsi, c'est bien sûr faire fausse route: il faudrait au contraire que les divers acteurs de la santé prennent ensemble des mesures efficaces. Toutefois, une telle démarche présuppose une culture du dialogue loyale et constructive.

«Si l'on y réfléchit, il est évidemment impensable qu'en ma qualité de président de la Fédération des médecins suisses (FMH), je salue des mesures préjudiciables aux médecins de familles, lesquels représentent déjà la catégorie de médecins soumise à la plus 
forte pression», explique J. de Haller. Hanspeter Kuhn, deuxième délégué de la FMH à la table ronde et juriste en chef de la FMH, confirme cette version des faits: «Au cours de cette rencontre, le président de la FMH a montré à quel point les médecins exerçant en cabinet ont déjà participé et participent encore à la réduction des coûts. Vu les signaux clairs et réitérés donnés par l'OFSP et le DFI à la FMH, indiquant que la baisse de la marge sur les médicaments avait été décidée depuis longtemps par la Confédération, le président de la FMH a relevé que cette mesure d'économie toucherait elle aussi le corps médical et pouvait donc être comptée parmi les contributions consenties par ce dernier en vue d'un allègement de la situation financière tendue du domaine de la santé. Mais cela ne signifie en aucun cas que la FMH approuve cette mesure d'économie supplémentaire, qui touchera une nouvelle fois principalement les médecins de premier recours et que la Confédération veut soi-disant soutenir.»

Le corps médical contribue déjà de manière déterminante à la baisse des coûts:

1. Au cours des dernières décennies, le revenu des médecins a baissé d'env. 40\% par rapport à celui d'autres professions.

2. Depuis l'introduction du TARMED en mai 2004 et après trois premières années de stricte neutralité des coûts, l'évolution de ces derniers dans les cabinets médicaux est strictement sous contrôle.

3. Avec la baisse des tarifs de laboratoire décidée récemment par le DFI, les médecins de premier recours subissent une nouvelle et grave diminution de leur revenu.

Lors de la table ronde, la FMH est partie de l'hypothèse, fondée sur les informations à disposition, qu'une baisse de $3 \%$ de la marge de distribution avait été décidée et serait mise en vigueur en 2009. On ne savait alors rien d'un abandon concret de cette mesure, que la FMH aurait pourtant vivement salué.

En septembre 2008, le président et le vice-président de la FMH ont adressé une lettre à Pascal Couchepin, alors président de la Confédération, pour lui demander des éclaircissements:

«[...] Avant les vacances d'été, l'Office fédéral de la santé publique nous avait informés que le prix des mé- dicaments chez les médecins et les pharmaciens subirait une baisse de trois pour cent au $1^{\text {er }}$ janvier 2009. Jusqu'ici, l'OFSP ne nous a toutefois fourni aucune base chiffrée justifiant cette réduction de $3 \%$ de la marge de distribution (baisse de 15 à $12 \%$ ). Cette décision est selon nous arbitraire, juridiquement infondée et uniquement motivée par des raisons politiques.

Nous nous adressons donc à vous, M. le Président de la Confédération, pour que vous renonciez à cette baisse de $3 \%$ du supplément sur les médicaments, et ce au nom de la satisfaction des patients et dans l'intérêt des médecins praticiens, de même que pour garantir l'approvisionnement à long terme tant en médicaments et qu'en soins médicaux. [...]».

La réponse du responsable du DFI du 27 octobre 2008 fut limpide:

«Il est donc fondamentalement justifié de baisser de $3 \%$ la marge de distribution. [...] En ce qui concerne la dispensation directe de médicaments, il existerait encore d'autres possibilités de réduction bien plus drastiques. Il convient néanmoins d'y renoncer pour l'instant, ne serait-ce que dans l'intérêt des médecins de premiers recours. Ainsi, il ne doit être appliqué qu'une baisse générale et pas de baisse différenciée de la marge de distribution [...]. Un entretien à ce propos ne se justifie pas pour l'instant [...]».

Après plusieurs contacts verbaux avec l'OFSP, la FMH a ensuite appris que cette mesure n'entrerait en vigueur qu'en mars, puis finalement à partir de juillet 2009 seulement. A aucun moment l'OFSP n'a signalé vouloir l'abandonner. C'est pourquoi la FMH l'a intégrée, bon gré mal gré, dans la liste des mesures d'économies devant être réalisées aux dépends du corps médical, ce qui ne signifie toutefois en aucun cas qu'elle l'approuve. «La démarche du DFI me déçoit et je regrette que le procès-verbal diffusé ait occasionné des malentendus, notamment chez les médecins de famille», souligne expressément Jacques de Haller. La FMH continuera à se battre fermement contre la baisse de la marge de distribution des médicaments et contre la dégradation de la médecine de premier recours. Le président de la FMH est en outre d'avis que cette démarche de Pascal Couchepin peut aussi être comprise comme une réaction de ce dernier face à la demande récente du corps médical exigeant sa démission. 